\title{
Identificação de bromato de potássio em pão do tipo francês na Cidade de Belém-
}

\section{Pará}

\author{
Identification of potassium bromate in bread of the french type in the City of Belém-Pará \\ Identificación de bromato de potasio en pan del tipo francés en la Ciudad de Belém-Pará
}

Recebido: 19/05/2021 | Revisado: 28/05/2021 | Aceito: 05/06/2021 | Publicado: 18/06/2021

\author{
Gleicy Kelly China Quemel \\ ORCID: https://orcid.org/0000-0003-1280-560X \\ Escola Superior da Amazônia, Brasil \\ E-mail: gkcquemel@gmail.com \\ Joel Vitor da Silva Goyzueta \\ ORCID: https://orcid.org/0000-0002-5887-745X \\ Escola Superior da Amazônia, Brasil \\ E-mail: jvgoyzueta@gmail.com \\ Luciana Pinheiro de Sousa de Assis \\ ORCID: https://orcid.org/0000-0001-5177-8910 \\ Escola Superior da Amazônia, Brasil \\ E-mail: lucianapinheiro1984@live.com \\ Talita Luiza da Silva Gonçalves \\ ORCID: https://orcid.org/0000-0003-1871-7643 \\ Escola Superior da Amazônia, Brasil \\ E-mail: talitaluizadasilva@yahoo.com.br
}

\begin{abstract}
Resumo
$\mathrm{O}$ bromato de potássio $\left(\mathrm{KBrO}_{3}\right)$ é um agente oxidante sintetizado a partir do bromo com o hidróxido de potássio, formando um sal com excelente resultado tecnológico e econômico quando utilizado na produção de pães, em particular do tipo francês, por agir no fortalecimento da cadeia do glúten sendo possível incorporar maior volume de ar na massa melhorando assim o resultado final. Porém, desde 2001 seu uso no Brasil foi proibido, pois descobriu-se que este sal possui elevado potencial carcinogênico e genotóxico. A análise do bromato de potássio é muito importante, pois fornece informações do resíduo bromato presente no produto final, devido a sua aplicação no setor da panificação que apesar de ilegal, ainda pode ser identificado, visto a vantagem do seu uso em relação às substâncias legalmente utilizadas com o mesmo propósito. Seja pela falta de fiscalização, desconhecimento da boa pratica de fabricação ou devido as leis brandas. Nesse sentido, este trabalho tem como objetivo identificar o $\mathrm{KBrO}_{3}$ em pães franceses na cidade Belém do Estado do Pará por meio de análise qualitativa experimental por método direto. Foram analisadas 20 (vinte) amostras aleatórias entre supermercados e padarias de diferentes bairros, das quais 17 (dezessete) apresentaram resposta positiva para a presença de bromato residual. Portanto, fica evidente que esse aditivo ainda faz parte da rotina de produção de pães do tipo francês colocando em risco a saúde do consumidor.
\end{abstract}

Palavras-chave: Bromato de potássio; Aditivo alimentar; Contaminação de pães.

\begin{abstract}
Potassium bromate $\left(\mathrm{KBrO}_{3}\right)$ is an oxidizing agent synthesized from bromine with potassium hydroxide, forming a salt with excellent technological and economic results when used in the production of bread, particularly of the French type, as it acts in strengthening the chain of gluten, being possible to incorporate a greater volume of air in the mass, thus improving the final result. However, since 2001, its use in Brazil has been banned, as it was discovered that this salt has a high carcinogenic and genotoxic potential. The analysis of potassium bromate is very important, as it provides information on the bromate residue present in the final product, due to its application in the bakery sector which, despite being illegal, can still be identified, given the advantage of its use in relation to substances legally used for the same purpose. Whether due to lack of inspection, lack of good manufacturing practice or due to soft laws. In this sense, this work aims to identify $\mathrm{KBrO} 3$ in French breads in the city of Belém, State of Pará, through experimental qualitative analysis using a direct method. Twenty (20) random samples from supermarkets and bakeries from different neighborhoods were analyzed, of which 17 (seventeen) showed a positive response for the presence of residual bromate. Therefore, it is evident that this additive is still part of the French-type bread production routine putting the consumer's health at risk.
\end{abstract}

Keywords: Potassium bromate; Food additive; Contamination of breads. 


\begin{abstract}
Resumen
El bromato de potasio $\left(\mathrm{KBrO}_{3}\right)$ es un oxidante sintetizado a partir de bromo con hidróxido de potasio, formando una sal con excelentes resultados tecnológicos y económicos cuando se utiliza en la elaboración de pan, particularmente del tipo francés, ya que actúa en el fortalecimiento de la cadena del gluten, siendo posible incorporar un mayor volumen de aire en la masa, mejorando así el resultado final. Sin embargo, desde 2001, su uso en Brasil está prohibido, ya que se descubrió que esta sal tiene un alto potencial cancerígeno y genotóxico. El análisis del bromato de potasio es muy importante, ya que proporciona información sobre el residuo de bromato presente en el producto final, debido a su aplicación en el sector de la panificación que, a pesar de ser ilegal, aún se puede identificar, dada la ventaja de su uso en relación a sustancias legalmente utilizadas para el mismo fin. Ya sea por falta de inspección, falta de buenas prácticas de fabricación o debido a leyes blandas. En este sentido, este trabajo tiene como objetivo identificar $\mathrm{KBrO} 3$ en panes franceses en la ciudad de Belém, Estado de Pará, a través de análisis cualitativo experimental utilizando un método directo. Se analizaron veinte (20) muestras aleatorias de supermercados y panaderías de diferentes barrios, de las cuales 17 (diecisiete) mostraron una respuesta positiva para la presencia de bromato residual. Por tanto, es evidente que este aditivo sigue formando parte de la rutina de producción de pan tipo francés poniendo en riesgo la salud del consumidor.
\end{abstract}

Palabras clave: Bromato de potasio; Aditivo alimentario; Contaminación de panes.

\title{
1. Introdução
}

O consumo do pão é uma pratica milenar e ao longo tempo a sua formulação e a forma de produção sofreram variações e adaptações. Seja industrial e/ou artesanal os processos foram se tornando cada vez escalonados e personalizados, reflexo de um mercado cada vez mais competitivo e empreendedor no setor de panificação (Sales, 2010).

Com isso a indústria passou a aplicar os aditivos, que são substâncias naturais ou artificiais que auxiliam na elaboração e/ou proporcionam melhor desenvolvimento da massa, que conferem melhores características para o pão como: textura, aroma, sabor, coloração, durabilidade. Neste contexto, o bromato de potássio passou a ser utilizado como aditivo na panificação por apresentar ação na cadeia do glúten, favorecendo um aumento no volume em $30 \%$, com melhoria na textura e granulação característica própria do pão do tipo francês (Machado et al, 2012).

Tal aplicação, despertou o interesse da comunidade cientifica que por sua vez, buscaram entender o impacto na saúde e na segurança do uso deste aditivo na aplicação na formulação dos pães. Os estudos realizados in vitro e in vivo, demonstraram o potencial cancerígeno e nefrotóxico do bromato de potássio $\left(\mathrm{KBrO}_{3}\right)$, o que levou a proibição, em qualquer quantidade, no Brasil (Dias et al, 2012).

Apesar do avanço das tecnologias e da proibição do uso, pela legislação brasileira com a Lei nº 10273/2001 (Brasil, 2001), ainda é possível identificar a comercialização de produtos pães com $\mathrm{KBrO}_{3}$ em diferentes estados, conforme os estudos científicos produzidos por Menezes et al (2007) que analisaram 78 amostras de melhoradores de pães e identificaram a presença bromato de potássio em 17,95\% desses melhoradores.

A problemática sobre a contaminação por bromato de potássio de origem alimentar afeta a sociedade como um todo, pois com base nos estudos toxicológicos que demostraram a grande relevância sobre questões ética, patológica e toxicológica que ainda precisam ser cuidadosamente tratadas pelas politicas públicas e órgãos fiscalizadores, tais com aplicação de ações fiscalizatórias mais eficientes e leis mais rígidas, visto a capacidade do bromato de potássio ser mutagênico, citotóxico e carcinogênico, tal como induzir tumores renais e tumores de células foliculares da tireoide (Kurokawa et al,1990)

Essa substancia induz inflamações moderada na mucosa gástrica que afeta o sistema nervoso central levando a surdez e espasmos, assim como patologias sistêmicas como: doença renal, hipotensão, cianose, metemoglobinemia e arritmia cardíaca, devido a citotoxicidade e toxicidade sistêmica. (Salami et al., 2020)

Portanto, causa sérios prejuízos à saúde pública, representando um grande impacto no orçamento publico com aumento de demanda judicial devido os tratamentos oncológicos terem um alto custo, pelo uso de componentes especializados, além de afetar diretamente o individuo biopsicossocial (Lima, 2012). 
Com base no exposto, foram realizadas analises qualitativas em pães do tipo francês produzidos e comercializados na cidade de Belém-Pará, a fim de identificar a presença de bromato de potássio pelo método direto, em triplicata, relacionar os dados com as bibliografias e discorrer sobre as relações patológicas.

\section{Metodologia}

O estudo é definido como uma pesquisa laboratorial com abordagem qualitativa, baseada na observação e interpretação do fenômeno e suas características conforme o meio em que se encontra (Pereira et al, 2018), e de amostragem não probabilística, que é baseada em critérios do pesquisador (Martins et al, 2016), de pães do tipo francês comercializados em diferentes regiões da cidade de Belém-PA, para avaliar a presença de bromato.

Foram coletados duas amostra de pães do tipo francês, aleatoriamente, em 20 estabelecimentos de diferentes bairros da cidade de Belém-Pará, sendo 17 (dezessete) padarias aleatórias e 3 (três) supermercados, no período de dezembro de 2021 a janeiro de 2021, adquiridas como consumidor nas padarias e supermercados. As amostras foram transportadas, sob condições assépticas, em sacolas de papel até o laboratório de bromatológica da Escola Superior da Amazônia (ESAMAZ). Posteriormente, foram enumerados para que o não fosse possível à identificação dos estabelecimentos.

O método utilizado para a análise de bromato residual foi o descrito por Emeje et al., (2010). No preparo da amostra, uma porção de 50g de amostra foi pesada, em balança analitica Gehaka AG200, e triturada no multiprocessador (marca de Walita de 650watts). Ultilizou-se $1 \mathrm{~g}$ de cada amostra e transferiu-se para um tubo falcon de $15 \mathrm{ml}$ juntamente $10 \mathrm{ml}$ de agua destilada. Em seguida, cada tubo falcon foi agitado no vortex, Phoenix AP 56, durante 20 minutos e centrifugado por 15 minutos a 3600rpm, na centrífuga Quimis de modelo Q222T116. Após a centrifugação o sobrenadante (5 ml) foi transferindo para um tubo de ensaio.

Para a determinação de $\mathrm{KBrO}_{3}$, na solução contida no tubo de ensaio, foi adicionando solução composta por KI 0,5\% (p/v) em $\mathrm{HCl}$ 0,1 mol/L. O aparecimento de uma coloração com tonalidade roxa a azul é confirmatória para bromato de potássio. Todas as análises foram realizadas em triplicata. Também foi utilizada uma amostra padrão como prova confirmatória com solução de $\mathrm{KBrO}_{3}$, com a adição da solução composta por $\mathrm{KI}$ 0,5\% (p/v) em $\mathrm{HCl}$ 0,1 mol/L, cuja coloração foi azul.

Cabe ressaltar que durante as análises e procedimentos foram realizados todos os cuidados de higiene e conservação, descrito por Feltes et al. (2016) nas analises bromatológica em alimento com a finalidade de evitar possível contaminação cruzada.

\section{Resultados e Discussão}

Após as análises, foi observado que das 20 (vinte) amostras apenas 03 (três) apresentaram resultado negativo e o restante das amostras, equivalente a 17 (dezessete) estabelecimentos, apresentaram resultado positivo para bromato em pão francês. Dentre as amostra positivas, 03 amostras eram de grandes redes de supermercado e 15 amostras de padarias localizadas em diferentes bairros de Belém, conforme observado na Tabela 1. 
Tabela 1: Identificação de bromato de potássio em panificadoras e supermercados por bairros na Região Metropolitana de Belém.

\begin{tabular}{|c|c|c|}
\hline Identificação & Bairro & Resultado \\
\hline P01 & Guamá & Positivo \\
\hline P02 & Jurunas & Positivo \\
\hline P03 & Marco & Positivo \\
\hline P04 & Castanheira & Positivo \\
\hline P05 & Coqueiro & Positivo \\
\hline P06 & Sacramenta & Negativo \\
\hline P07 & Batista Campos & Positivo \\
\hline P08 & Canudos & Positivo \\
\hline P09 & Umarizal & Positivo \\
\hline P10 & Sideral & Positivo \\
\hline P11 & Pedreira & positivo \\
\hline P12 & Reduto & Negativo \\
\hline P13 & Cremação & Negativo \\
\hline P14 & Campina & positivo \\
\hline P15 & Nazaré & Positivo \\
\hline P16 & Cabanagem & Positivo \\
\hline P17 & Marex & Positivo \\
\hline S01 & Souza & Positivo \\
\hline S02 & Parque verde & Positivo \\
\hline S03 & Terra firme & Positivo \\
\hline
\end{tabular}

P: Amostra de padaria, S: Amostra de supermercado. Fonte: Autores (2021).

O resultado porcentual das amostras contaminadas por bromato de potássio residual foi de $85 \%$ (figura 1), o que confirma o uso desse adulterante na produção de pães do tipo francês, o que é considerado alarmante devido o alto risco de intoxicação para a saúde tanto da população residente da cidade de Belém quanto para os demais municípios da região metropolitana. Além disso, o consumo elevado de pão contaminado, por hábitos culturais na região colabora visto que uma quantidade mínima de bromato de potássio é suficiente para causar diferentes níveis danos a saúde. 
Figura 1: Percentual de amostra contaminada e não contaminada.

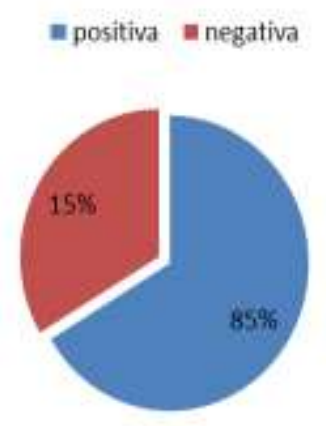

Fonte: Autores (2021).

No Brasil apesar de existir outros agentes oxidantes legalizados, pode-se observar que até o presente momento o uso de $\mathrm{KBrO}_{3}$ ainda é utilizado na maioria de estabelecimento que comercializam e produzem os pães, especialmente o pão francês, conforme foi identificado nas amostras analisadas na cidade de Belém do Pará, o que não difere muito de outros Estados do Brasil , como pode ser observado na Tabela 2.

Tabela 2: Percentual de amostra positiva para $\mathrm{KBrO}_{3}$ por Estado no Brasil.

\begin{tabular}{c|ccc}
\hline Estado & Percentual positivo & Ano & Referencia \\
\hline PA & $85 \%$ & 2021 & Autor \\
\hline SC & $3 \%$ & 2019 & Luciano et al. \\
\hline RO & $0 \%$ & 2018 & Lima et al. \\
PI & $100 \%$ & 2012 & Carvalho et al. \\
RN & $24 \%$ & 2014 & Queiroz et. al. \\
& $60 \%$ & 2015 & Melo et. al. \\
CE & 41,7 & 2008 & Santos, \\
\hline
\end{tabular}

Fonte: Autores (2021).

Cabe ressaltar que assim como no Brasil outros países também proíbem o uso de bromato como na União Europeia e Reino Unido, banido em 1990; na Nigéria, banido em 1993; no Canadá, banido em 1994; no Peru e Colômbia, ambos banidos em 2002; e na China e Líbia, banido em 2005(Rana et al, 2020).

Nos estudos de Johnson et al. (2013) foram analisadas amostras de pão e farinha da cidade de karu, no estado de Nasarawa localizado no norte de Nigéria. No pão, as amostras analisadas variaram entre $0,5 \mu \mathrm{g} / \mathrm{g}$ a $8,4 \mu \mathrm{g} / \mathrm{g}$ e na farinha de trigo $0,83 \mu \mathrm{g} / \mathrm{g}$ a $1,42 \mu \mathrm{g} / \mathrm{g}$, logo continham bromato de potássio em grande quantidade.

Já em 2019, Aletan (2020) também avaliou os níveis de bromato de potássio do pão vendido e consumido em Lagos e nos subúrbios, na Nigéria, e os resultados revelaram que os padeiros não cumprem a proibição do bromato de potássio estipulada tanto pela Agência Nacional de Controle e Administração de Alimentos e Medicamentos (NAFDAC) quanto pela OMS (Organização Mundial de Saúde).

Os estudos realizados na cidade de Jaipur, na Índia, revelou que os níveis de bromato variaram de 10,72 \pm 3.75 ppm na amostra de pão branco a 39,73 $\pm 5,32$ ppm no pão de frutas, o que mostram que as padarias não estão seguindo os padrões 
adequados de preparação do pão. Já em amostras de pão branco e marrom de apenas algumas marcas o bromato não foi detectado, o que indicou que o processo de fabricação do pão foi adequado para essas marcas (Rana et al, 2020).

Na cidade de Tabouja, na Líbia, o pão é altamente consumido por todos os grupos socioeconômicos; isto é consumido principalmente no café da manhã, jantar e merenda escolar. Ao avaliar o pães, foi também observado que $100 \%$ das anta testadas continham altos níveis de bromato de potássio residual. (Alhanash et al, 2020).

No sentido oposto a proibição, os Estados Unidos permite o uso de Bromato de potássio até $75 \mathrm{mg} / \mathrm{kg}$, porém são realizadas campanhas para a diminuição do uso pelos fabricantes de farinha e padarias. (Food and drug administration, 2021)

De acordo com Pereira et al. (2009), que avaliaram a influência de vários agentes oxidantes, destacou-se o ácido ascórbico como um substituto legal, pois apresentou bons resultados tecnológico na produção de pães, quando comparado ao $\mathrm{KBrO}_{3}$,

Quanto aos riscos, às evidências da relação patológica do bromato de potássio, se dá pelos ensaios realizados em animais, principalmente em ratos, que apontarampotencial toxicológico, mutagênico e nefrotóxico, descritos na Tabela 3.

Alguns casos de intoxicação foram registrados ao longo do tempo, desse modo foi estimada a dosagem letal para humanos adulto na faixa de $5 \mathrm{mg} / \mathrm{kg}$ a $500 \mathrm{mg} / \mathrm{kg}$ por peso corporal com uma taxa de mortalidade de $54 \%$ e sintomas como vomito, diarreia, dor abdominal, oliguria, anúria, trombocitopenia, vertigem e depressão (Starek \& Starek-Świechowicz, 2016).

Também há registro de casos de intoxicação acidental em crianças onde a dosagem letal estimou-se entre 20mg a $1 \mathrm{~g} / \mathrm{kg}$ de peso corporal. Os principais sintomas apresentados nas crianças foram sedação, letargia e depressão do sistema nervoso central, assim como a evidente toxicidade renal que evoluíram para insuficiência renal em 36 casos relatados, comprovado através de exames de biópsias indicando edema e fibrose intersticial, atrofia tubular e separação epitelial dos túbulos proximais (Kumar \& Pankaj, 2012).

Tabela 3: Toxicidade do bromato de potássio nos órgãos.

\begin{tabular}{ccc}
\hline Órgão & Experimento animal & Referencia \\
\hline Alteração da função hepática & Rato albino suíço & Hassan I et al., 2019 \\
Toxicidade hepática & Ratos wistar & Awoniran PO and Adeyemi DO, 2018 \\
Alteração no cerebelo & Rato adulto & Ben Saad et al., 2018
\end{tabular}

Toxicidade renal e hepática Rato suíço Altoom et al., 2018, Kujawska etal 2013

\begin{tabular}{lll} 
Toxicidade hepática & Rato adulto albino & Bayomy et al., 2016 \\
\hline Alteração neurocomportamento & Rato suíço & Ajarem et al., 2016 \\
Toxicidade no osso e sangue & Ratos adultos & Ben Saad et al., 2015 \\
\hline Toxicidade Cardíaca & Rato albino macho & El-Deeb and Abd-El-Hafez., 2015 \\
Toxicidade renal & Ratos F344 & Dodd et al., 2013, \\
\hline
\end{tabular}

Fonte: Adaptado de Shanmugavel (2019) 
O bromato de potássio é considerado um potencial carcinógeno humano pela Agência Internacional de Pesquisa sobre o Câncer (IARC) e classificado na classe 2B, isto é, quando existem evidências limitadas de que o agente é carcinogênico para humanos e evidências suficientes de que ele é carcinogênico para animais ou quando não há evidências suficientes em ambos os casos, mas há dados relevantes de que ele possa ser carcinogênio. Por isso, países em todo o mundo o baniram parcial ou totalmente o bromato de potássio (Shanmugavel et al, 2020).

$\mathrm{O}$ uso de $\mathrm{KBrO}_{3}$, apesar de proibido por lei, com possíveis aplicações de sanções penais a quais podem chegar até a 15 anos de prisão, ainda está presente nos pães comercializado no país, apesar dos estudos realizados nacional e internacionalmente, não apontam de que forma a adulteração acontece, se de forma direta com pratica ilícita por parte dos padeiros ou de forma indireta por aquisição de melhoradores ou insumos adulterados. O que se faz necessário são fiscalizações efetivas e qualificadas dos órgãos responsáveis tanto nas padarias quanto nas indústrias (Albino, 2006).

Já que uma das medidas que podem ser consideradas preventivas e protetivas referem-se à efetiva fiscalização dos órgãos competentes responsáveis por executar medidas de segurança voltada para a proteção dos consumidores, pois o consumo de alimento adulterado pode causar várias complicações o que representa uma ameaça à saúde da sociedade, infligindo medo e insegurança à população e como consequência prejuízos diversos e a perda de confiança e credibilidade nas autoridades governamentais responsáveis pela segurança alimentar no país (Figueira, 2018).

Uma alternativa de consumo a qual tem o apelo para a saudabilidade é o consumo de pães produzindo com outro tipo de farrinha como: farinha de sorgo, de arroz, de banana, de cevada, de ervilha e de chia, que podem proporcionar o melhoramento de características sensoriais, funcionais, nutricionais, a fim de trazer benefícios ao consumidor (Oliveira et al, 2020)

Além disso, é necessária a realização de campanha educacional e preventiva sobre o risco da adulteração em alimento com maior amplitude seguindo as orientações feitas especialmente pelo o Comitê Conjunto da OMS de Peritos em Aditivos Alimentares (JECFA). Outra medida seria melhorar a qualidade e segurança na indústria de alimentos, em destaques os programas de Boas Práticas de Fabricação (BPF) e análise de Perigos e Pontos Críticos de Controle (APPCC), ambas são ferramentas recomendadas pelos órgãos de fiscalizações, tais como agencia Nacional de Vigilância Sanitária (ANVISA) e Ministério da Agricultura Pecuária e Abastecimento (MAPA), sendo recomendado em toda a cadeia produtiva (Vieira, 2019).

Sobre a adulteração, como é o caso do uso de bromato de potássio no pão em países proibidos, se faz necessário entender o conceito Ford and Drug Administration que de acordo com Wurlitzer (2007) é um conceito bastante abordado em países desenvolvidos que visam ações relacionadas com a prevenção de atos criminosos em alimentos para a proteção da cadeia produtiva de alimentos contra atos intencionais ou não de contaminação ou adulteração. Cabe ressaltar que alterar um produto com substância proibida é considerado crime hediondo de acordo com artigo 272 do Código Penal Brasileiro, com pena de reclusão de 10 a 15 anos e multa. (Brasil, 1940)

\section{Conclusão}

O presente trabalho chama a atenção para os resultados obtidos nas analises, pois dentre as 20 amostras analisadas na cidade de Belém- PA, 15\% resultaram em negativo, totalizando $85 \%$ das amostras contaminadas Haja vista não se admitir nenhum percentual pela legislação atual.

No Brasil as legislações que tratam sobre a temática do alimento, tais como a lei de aditivos e Boa Pratica de Produção (BPF), as quais visando garantir níveis aceitáveis de segurança, qualidade e controle sanitário. Para exemplificar sobre este assunto recorrem-se as propostas voltadas para melhorar a qualidade e segurança na indústria de alimentos, em destaques os programas de Boas Práticas de Fabricação (BPF) e análise de Perigos e Pontos Críticos de Controle (APPCC), ambas são ferramentas recomendadas pelos órgãos de fiscalizações, tais como agencia Nacional de Vigilância Sanitária 
(ANVISA) E Ministério da Agricultura Pecuária e Abastecimento (MAPA), sendo recomendado em toda a cadeia produtiva.

Uma das medidas que podem ser importantíssima é a efetiva fiscalização dos órgãos competentes responsáveis por executar medidas de segurança voltada para a proteção dos consumidores, pois o consumo de alimento adulterado pode causar danos à saúde, até mesmo a morte. Porém, observa-se que certos assuntos são negligenciados há anos e se tratando da temática deste trabalho já se passaram décadas deste a proibição legal do uso do bromato de potássio no Brasil. Cenário como este admitisse o aumento de oportunidades de trabalho para os diversos profissionais correlacionados a área de alimentos, se devidamente qualificados, isto é: capacitados para o trabalho em equipe multiprofissional, onde haverá a complementariedade de conhecimentos e práticas. Sendo estes possuidores de conhecimentos técnico- científicos nas áreas da ciência e tecnologia de alimentos bem como a participação mais ativa nos processos de "como fazer a qualidade" que nos tradicionais de "como a qualidade" assim como na fiscalização ativa.

Portanto, fica evidente a necessidade de mais estudos voltados à identificação e rastreio quanto a origem desta substancia usada na adulteração, assim como identificação do bromato de potássio nos produtos finais de panificação, uma vez que o uso desse agente oxidante ainda se faz presente em vários Estados do Brasil mesmo sendo proibido.

\section{Referências}

Albino, E., Gomes, S. C., Santos, A. C. O. \& Beltrão, A. B. (2009). Bromato de potássio em aditivos para a panificação usada em padarias do III Distrito Sanitário do Recife, PE-2006. Hig Alim. 23(170/171), 151-5.

Aletan, U.I. (2020) Potassium Bromate Content of Bread Samples in Lagos City. Nigerian Journal of Pharmaceutical and Applied Science Research. 8(2), 3336.

Alhanash, H. B., Edriss, N. A., Ksheid, S. M. \& Issa, R. A. M. (2020). Residual Bromate Assessment in Bread Samples from Tajouracity bakeries, Libya. Academic Journal of Research and Scientific Publishing, 2(13).

Brasil. (1940). Executivo. Decreto-lei No 2.848, de 7 de dezembro de 1940. Código Penal. https://www.planalto.gov.br/ccivil_03/decreto-lei/del2848.htm.

Brasil. (2001). Lei $n^{\circ}$. 10.273, de 5 de setembro de 2001. Dispõe sobre o uso do bromatode potássio na farinha e nos produtos de panificação. Diário Oficial da União. 1.

Dias N. A., Martins, M. S. \& Lichtig, J. (2012) Identificação de íons brometo e bromato em preparados para produtos de panificação Bol Inst Adolfo Lutz. 22(1):12-14.

Emeje, M. O., Ofoefule, S. I., Nnaji, A. C., Ofoefule, A. U. \& Brown, A. S. (2010). Assessment of bread safety in Nigeria: Quantitative determination of potassium bromate and lead. African Journal of Food Science. Nigéria, 4 (6), 394 -397.

Feltes, M. M. C. et al. (2016). Procedimentos Operacionais Padronizados de Bromatologia de Alimentos. Instituto Federal Catarinense.

Figueira, L. C. (2018). Os Conceitos de defesa dos alimentos e Fraude emAlimentos aplicados em Fabricas de Temperos Carneos. Um estudo de Caso. Dissertação. Faculdade de Zootecnia e Engenharia de Alimentos - Universidade de São Paulo.

Food Additive Status List: Potassium bromate. (2021). https://www.fda.gov/food/food-additives-petitions/food-additive-status-list.

Johnson, O. R., Daniel, K. D., Iyabo, A. G. G. \& Oloruntoba, A. C. (2013) Analysis of Potassium Bromate and Hydrocyanic Acid Contents of Commonly Consumed Loaves of Bread and Wheat Flour Samples In Karu,Nasarawa State, Nigeria. Journal Of Environmental Science, Nigeria, 6 (7), $42-46$.

Kumar, S. \& Pankaj, P. (2012). Intoxicação acidental por bromato de potássio em nove adultos. Journal of Indian Academy of Forensic Medicine, [S. l.], 364366.

Kurokawa, Y., Maekawa,A., Takahashi, M. \& Hayashi, Y (1990). Toxicity and Carcinogenicity of Potassium Bromat: A New Renal Carcinogen. Environmental Health Perspectives, 87. 309- 335

Lima-Dellamora, E. C., Caetano, R. \& Osório-de-Castro, C.G.S. (2012). Dispensação de medicamentos do componenteespecializado em polos no Estado do Rio de Janeiro. Saúde coletiva, Rio deJaneiro. 17(9), 2387-2396,

Luciano, E. A., Amaral,E. L., Silva, F. C. M., Martins, P. J. \& Hartin, SD (2019). A utilização do bromato de potássio no setor da panificação industrial. Monografia (Curso Técnico em Química) - Instituto Federal Catarinense, Araquari.

Machado, A. et al. (2012). Guia de boas práticas nutricionais pão francês.Brasilia: Agência Nacional de Vigilância Sanitária.1(1), 24. 
Martins, A. M., Gesser, G. A. \& Machado, L. E. (2016). Análise do perfil metodológico das dissertações de mestrado profissional em administração universitária da universidade federal de santa catarina apresentadas no período de 2012 a 2015. In: XVI Colóquio internacional de Gestion Universitaria, Arequipa, Peru.

Menezes C. R. B et al, (2007). Pesquisa de bromato de potássio em produtos de panificação. In: Anais do XV Encontro Nacional de Análises de Alimentos.

Melo, V. et al. (2015). Análise Da Presença De Bromato De Potássio Em Pães Produzidos E Comercializados Em Panificadoras Do Município De Mossoró RN. Higiene Alimentar 29, 248-249.

Oliveira, I., Melo, F. S. N., Sousa, M. M., Paz, E. O. \& Cavalcanti, M. S. (2020). Utilização de farinhas alternativas em produtos de panificação: uma revisão literária. Research, Society and Development, 9 , e441996228

Pereira, A. S., Shitsuka, D. M., Parreira, F. J. \& Shitsuka, R. (2009) Influência de agentes oxidantes sobre as propriedades reológicas de massas de farinha de trigo branca e de grão inteiro e sobre o volume específico de pão francês. Brazilian Journal of Food Technology, Brasil, ano 3, 12, 161-171, 1.

Queiroz, D. J. M., Moura, E. V. \& Cunha, M. A. (2014). Avaliação da contaminação por bromato em pães do tipo francês. Revista Instituto Adolfo Lutz, Rio Grande do Norte, 233-237.

Rana, R., Mehtani, P. \& Sharma, C. (2020). Analysis of potassium bromate in bread samples of Jaipur City, India. Annals Food Science and Technology, Mansarovar, 21, 109-116.

Salami, A. T., Adebimpe, M. A., Olagoke, O. C., Iyiola, T. O. \& Olaleye, S. M. (2020) Potassium bromate cytotoxicity in the Wister rat model of chronic gastric ulcers: Possible reversal by protocatechuic acid. J FoodBiochem 44:e13501.

Sales, S. (2010). O Culto do Pão. Dissertação (Mestre em Animação Artística) - Instituto Politécnico de Bragança, Escola Superior de Educação.

Santos, R. G. (2008). Analise Quantitativa de Bromato no Pao Frances Comercializado na Cidade de Fortaleza. Trabalho de Conclusão de Curso (Graduação em Farmácia - Habilitação em Bromatologia) - Universidade Federal do Ceará.

Sousa, L. F. S. \& Carvalho, L. F. M. (2012). Determinação de bromato de potássio em pão francês comercializados em Teresina - pi. In: Congresso Norte Nordeste de pesquisa e inovação, Palmas, p. 1-4.

Starek, A. \& Starek-Świechowicz, B. (2016). Toxicological Properties of Potassium Bromate. Journal of Pharmacological Reports, 1 (3), 1-9, 15.

Vieira, V. B. (2019). Analise de Perigos e Pontos Críticos de Controle: Benefícios e etapas. 2019. https://news.certifee.com.br/artigo/Analise-de-Perigos-ePontos-Criticos-de-Controle-APPCC-Beneficios-e-etapas.

Wurlitze, R. N. J. et al. (2018). Optimization of enzymatic treatment to produce yacon juice clarified by microfiltration with high levels of chlorogenic acid and fructooligosaccharides. Journal of food processing and preservation. 42, e13641. 